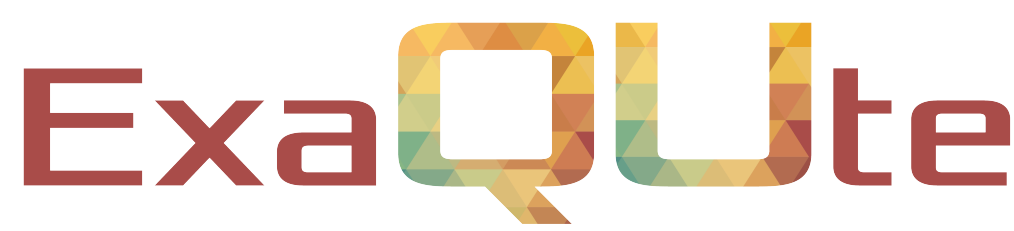

Exascale Quantification of Uncertainties for

Technology and Science Simulation

\title{
D5.1 ExaQUte API for MLMC
}

\section{Document information table}

\begin{tabular}{|l|l|}
\hline Contract number: & 800898 \\
\hline Project acronym: & ExaQUte \\
\hline Project Coordinator: & CIMNE \\
\hline Document Responsible Partner: & TUM \\
\hline Deliverable Type: & Report, Other \\
\hline Dissemination Level: & Public \\
\hline Related WP \& Task: & WP5, Task 5.1 \\
\hline Status: & Final version \\
\hline
\end{tabular}

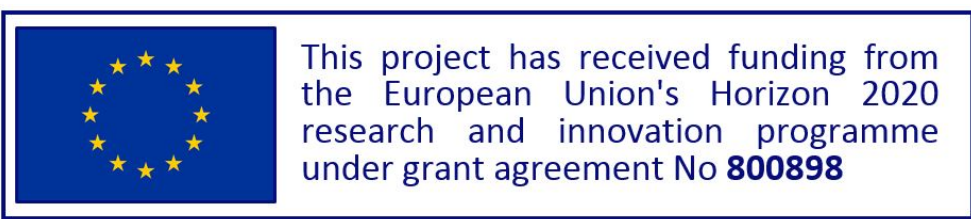




\section{Authoring}

\begin{tabular}{|l|l|l|l|l|}
\hline Prepared by: & Partner & Modified Page/Sections & Version & Comments \\
\hline Authors & TUM & & & \\
\hline Daniel Drzisga & TUM & & & \\
\hline Mario Teixeira Parente & TUM & & & \\
\hline Roland Wüchner & TUM & & & \\
\hline Contributors & & & & \\
\hline & & & & \\
\hline & & & & \\
\hline
\end{tabular}

\section{Change Log}

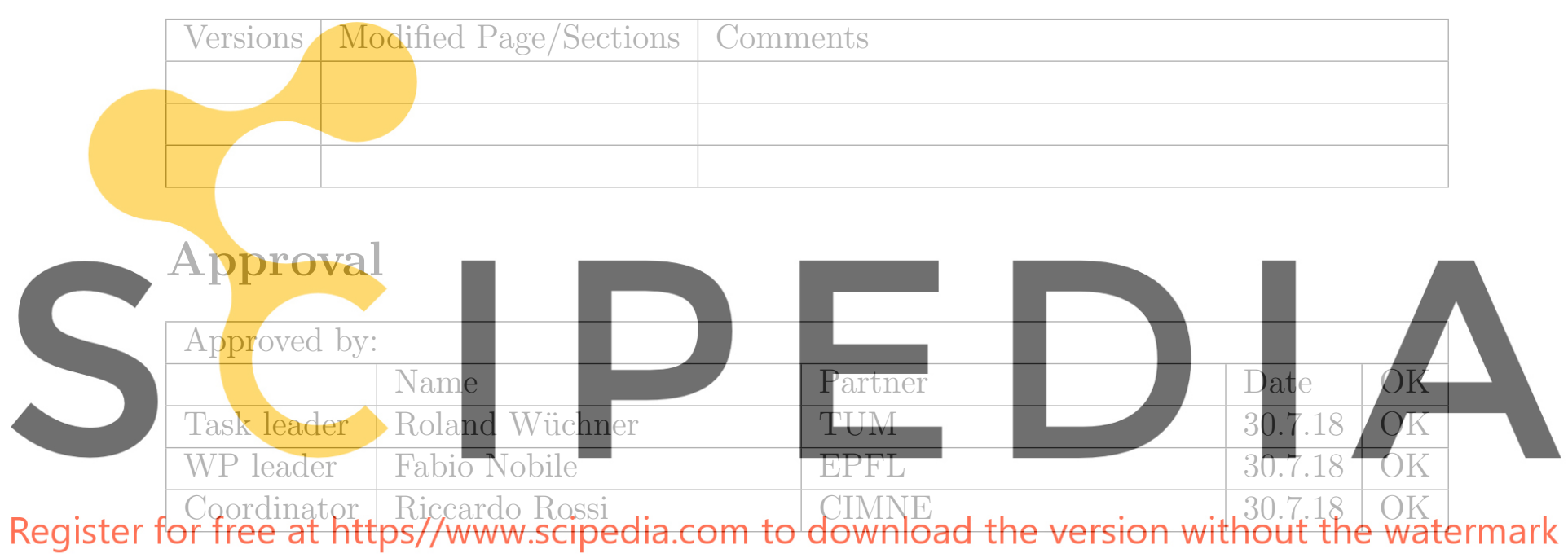




\section{Executive summary}

This deliverable focuses on the design of an interface between MLMC algorithms, the scheduling engine, and problem solvers. For this purpose an API definition is proposed together with a basic reference implementation in Python. This work serves as a first step for the development of the ExaQUte MLMC Python engine.

It includes:

- API definition

- Demonstrator code and description

- Example of usage
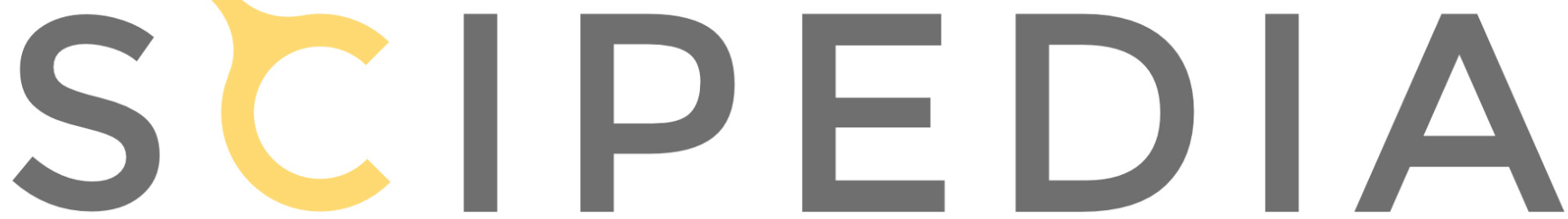

Register for free at https//www.scipedia.com to download the version without the watermark 


\section{Table of contents}

1 Introduction $\quad 8$

2 API definition $\quad 8$

2.1 Problem Abstraction . . . . . . . . . . . . . . . . . . 8

2.1.1 MLMC Algorithm interface . . . . . . . . . . . . . . . . . 9

2.1.2 Problem interface . . . . . . . . . . . . . . . . . . . . . 10

3 Demonstrator code $\quad 10$

3.1 MLMC Algorithm implementation . . . . . . . . . . . . . . . . 10

3.2 Elliptic benchmark-problem implementation . . . . . . . . . . . . . 10

4 Example of usage

A Snippets
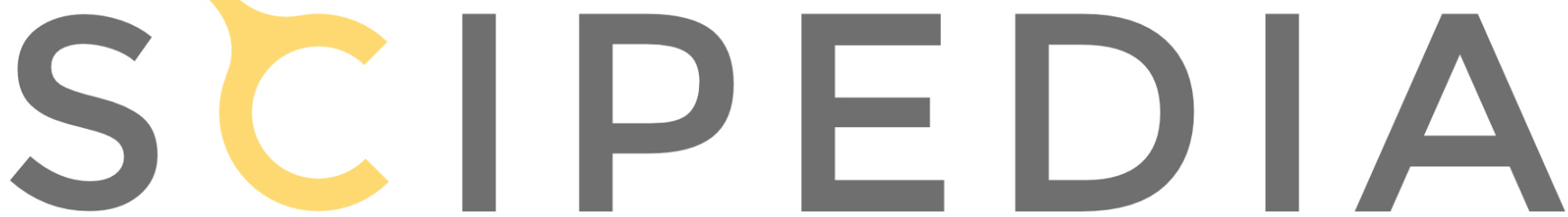

Register for free at https//www.scipedia.com to download the version without the watermark 


\section{List of Figures}

1 Schema of our MLMC API with three solver levels running in parallel. . . 9

2 Example MLMC settings class encapsulating all the required MLMC parameters for a particular MLMC strategy. In this case the C-MLMC algorithm. . . . . . . . . . . . . . . . . . 13

3 Example implementation of the problem interface. It encapsulates information of the problem, the solver, as well as the QoI. . . . . . . . . . . . 13

4 Example implementation of the MLMC core interface. In this particular case, the C-MLMC algorithm is implemented. (part 1) . . . . . . . . . . . 14

4 Example implementation of the MLMC core interface. In this particular case, the C-MLMC algorithm is implemented. (part 2) . . . . . . . . . . 15

5 Example program where an MLMC settings and a Problem class are instantiated and the MLMC algorithm is executed. The MLMC algorithm internally instantiates problem solver processes with respective mesh resolutions depending on the underlying MLMC strategy. In this particular example, the C-MLMC algorithm is used. .
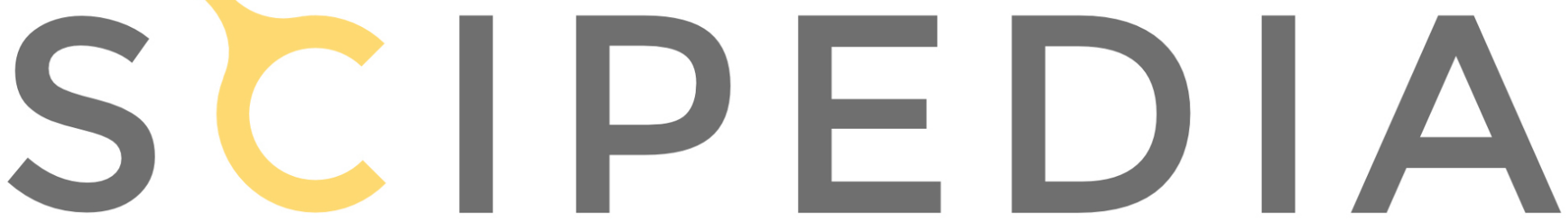

Register for free at https//www.scipedia.com to download the version without the watermark 


\section{List of Tables}

1 Number of samples per level $L$ adaptively chosen in nine steps. . . . . . . . 11
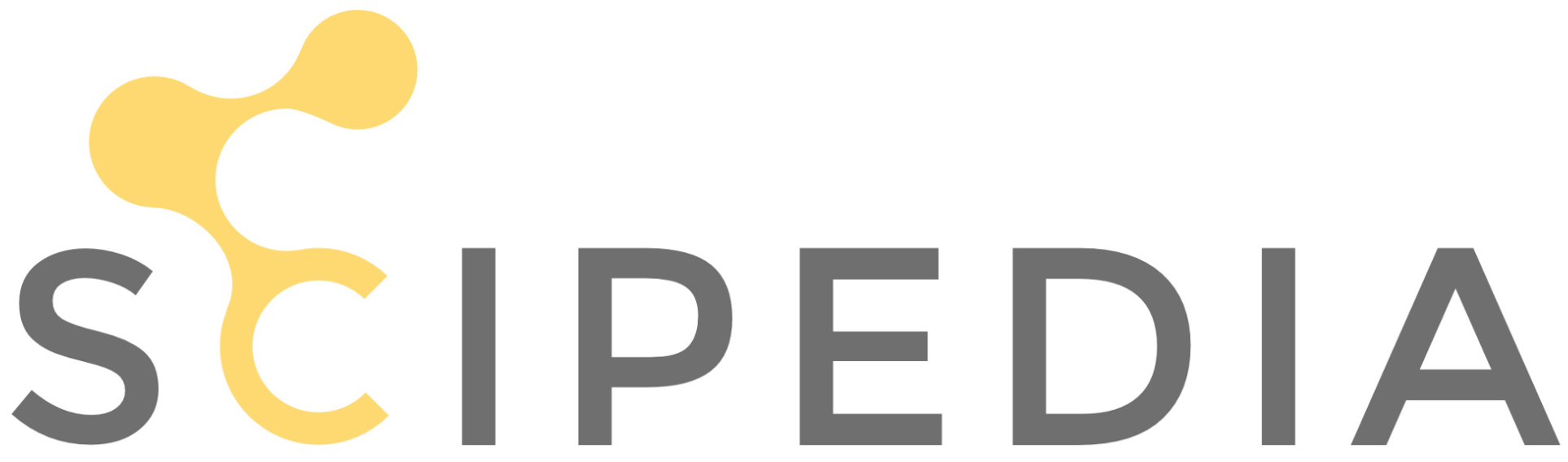

Register for free at https//www.scipedia.com to download the version without the watermark 
Nomenclature / Acronym list

\begin{tabular}{|l|l|}
\hline Acronym & Meaning \\
\hline \hline API & Application Programming Interface \\
\hline ExaQUte & $\begin{array}{l}\text { EXAscale Quantification of Uncertainties for Technology } \\
\text { and Science Simulation }\end{array}$ \\
\hline QoI & Quantity of Interest \\
\hline MC & Monte Carlo \\
\hline MLMC & Multilevel Monte Carlo method \\
\hline C-MLMC & Continuation Multilevel Monte Carlo method \\
\hline HPC & High performance computing \\
\hline PDE & Partial differential equation \\
\hline
\end{tabular}
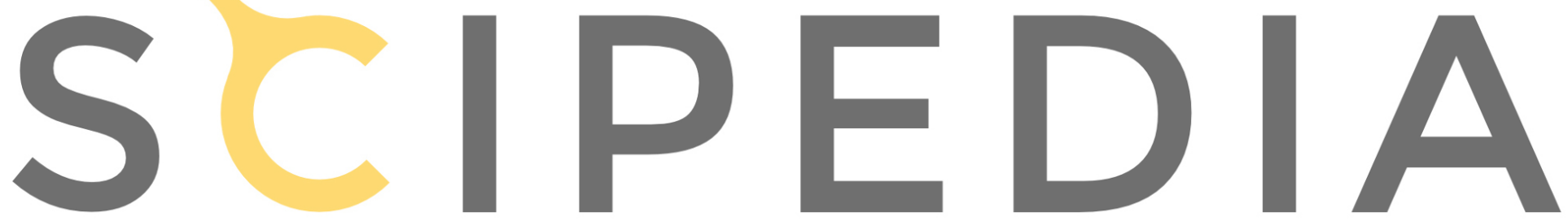

Register for free at https//www.scipedia.com to download the version without the watermark 


\section{Introduction}

In this report, we give a brief introduction to the MLMC method and its main goals; we also provide a first API definition of the MLMC Python engine in Section 2 and a reference implementation for a test problem in Section 3.

The Multilevel Monte Carlo method (MLMC) $[1,5,6,8]$ is a technique to reduce computational cost for quantifying uncertainties of a random output Quantity of Interest (QoI) of a complex computational model. It estimates statistics of the QoI like e.g., its expected value. Let $Q$ denote the output QoI which is random due to randomness in the input parameter of the model. We are interested in computing, or more precisely, approximating $\mathbb{E}[Q]$. MLMC is flexible since it does not require regularity of the parameter-to-QoI map and furthermore breaks the curse of dimensionality, i. e., its performance does not depend on the number of input random parameters. MLMC accelerates convergence of estimators of $\mathbb{E}[Q]$, with respect to standard Monte Carlo (MC) estimators, which are often unaffordable for complex computational models. Also, it is well-suited for parallelization in high performance computing (HPC) contexts [3].

Informally, MLMC distributes computational work on different levels from a hierarchy of different accuracies, e. g,. a hierarchy of meshes for approximating solutions of partial differential equations (PDEs). A crucial decision using MLMC is how much work is allocated to each level and how the overanl error is split into-different contributions.
Standard MLMC splits the overall error into two equal parts, related to the discretization
of the PDE and the statistical errors of the level-wige MC estimators, although different
splitings are also possible [7].
Also, the strategy of distributing the work over the levels relies on approximations of
variances, which can be expensive to compute. There exist adaptive versions of MLMC that choose optimal values for the number Register for free at https//www. scipedia.com to download the version without the watermark for computing optimal values should not exceed the overall cost of the resulting MLMC estimator. A recent variant of MLMC, tackling these issues, is the Continuation Multilevel Monte Carlo method (C-MLMC) [2, 9], which uses an algorithm to learn parameters for distributing the work and splitting the overall error accordingly, in order to save as much cost as possible while guaranteeing a final error within a prescribed tolerance.

\section{API definition}

In this section, we propose an API for the development of an ExaQUte MLMC Python engine. For this purpose, we follow a modular approach where the MLMC algorithm is completely decoupled from the problem to be solved. This allows for rapid application development of different MLMC strategies without having to change anything in the problem solver and vice versa. The presented API and the reference implementation in Section 3 are only a first proposal and subject to change in the future.

\subsection{Problem Abstraction}

In order to decouple the problem of interest from the employed MLMC algorithm, we propose the following two interfaces. First, the $M L M C$ Algorithm interface is described in Section 2.1.1 and then the Problem interface is described in Section 2.1.2. 
Figure 1 shows a schematic view of our API where the MLMC algorithm spawns new solver processes for each required sample and after a task is finished, receives the corresponding QoI of a sample. In this particular example, solvers on three levels are running in parallel. Solvers on finer levels require more computational resources, i.e., more memory and larger number of processors. Since the goal of the MLMC method is to do most of the work on coarse levels, most of the samples are only required to be computed on the coarse levels.

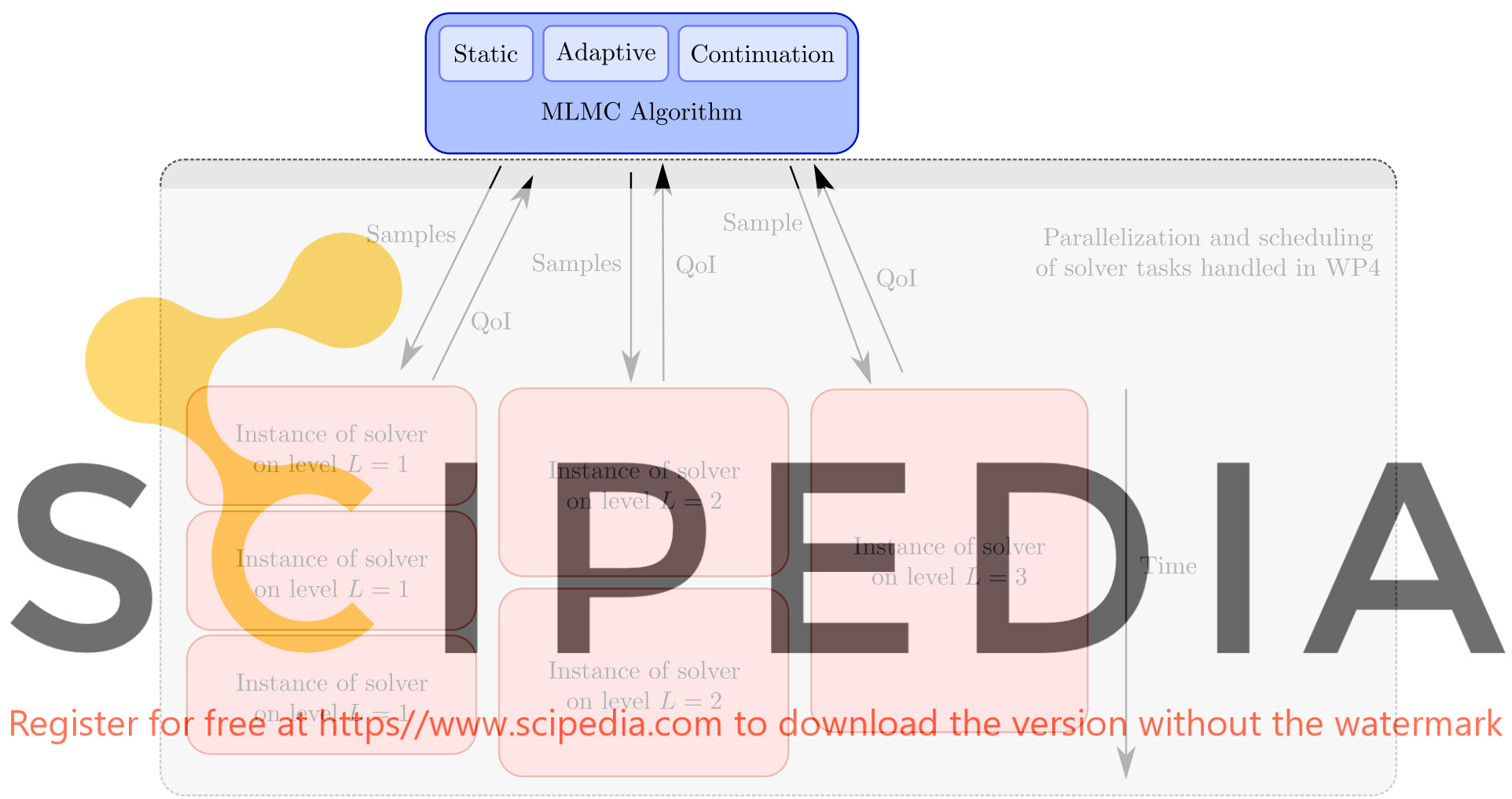

Figure 1: Schema of our MLMC API with three solver levels running in parallel.

\subsubsection{MLMC Algorithm interface}

Implementations of the MLMC algorithm interface are the core of each MLMC simulation (cf. blue box in Figure 1). A particular implementation is responsible for the distribution of samples for each level and the computation of statistics of the QoI. As described in Section 1, it is possible to implement different strategies and we mainly focus on static, adaptive, and continuation strategies.

Each instance of the MLMC algorithm requires information on the problem which needs to be solved. This is done through the Problem interface described in Section 2.1.2. This interface provides a method that returns the QoI obtained on a particular level. At each MLMC iteration, the algorithm decides how many samples are required on each level such that a given accuracy is obtained. The samples are then passed to the scheduler which is then responsible for the distribution of tasks depending on the required resources (cf. gray box in 1). After a task finishes, the MLMC algorithm receives its QoI. Depending on the MLMC strategy and the problem, further iterations may be necessary before the algorithm converges. 


\subsubsection{Problem interface}

The Problem interface encapsulates information of the problem of interest, its QoI, and the underlying solver. It provides a method which returns the QoI obtained on a specific level. This method is called either by the MLMC algorithm directly or its attached scheduler for each sample. In our typical cases, calling this method involves solving a PDE with a randomly sampled coefficient on a refined mesh.

The red boxes in Figure 1 show different instances of Problem interface implementations running in parallel. It is important that the implementations of this interface must be thread-safe and may not interfere with other instances when executed in parallel.

Moreover, the solvers themselves may run in parallel which has to be taken into account by the task scheduler, so that the provided computational resources are efficiently utilized.

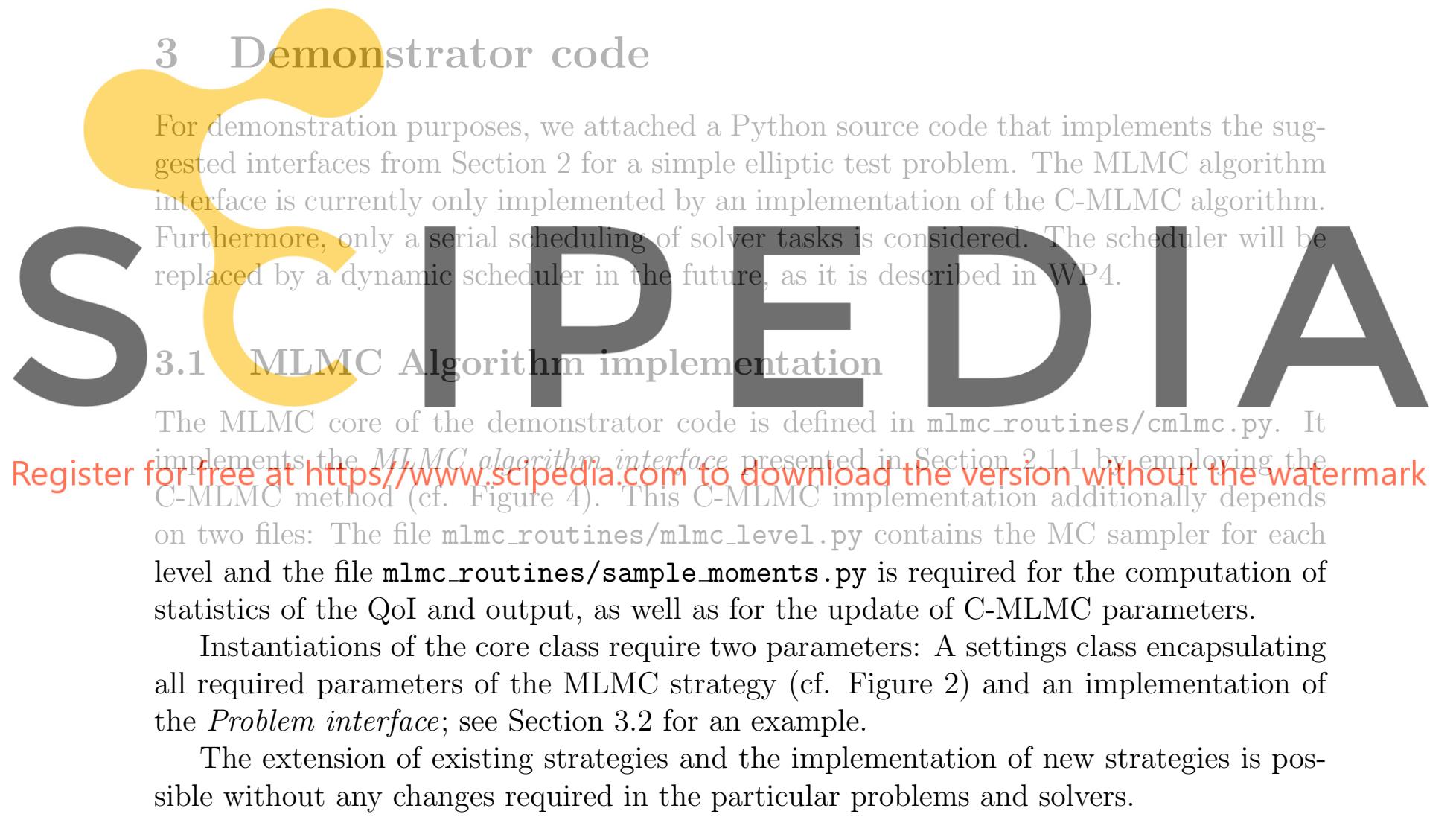

\subsection{Elliptic benchmark-problem implementation}

The class ellipt_2d (cf. Figure 3) implements the Problem interface described in Section 2.1.2. It is found in the file benchmark_problems.py and implements the discretization, solver, and QoI computation of the following elliptic benchmark problem in 2D [8, Section 5.2]:

Let $D:=[0,1]^{2}$. Find $u \in C^{2}(D) \cap C(\partial D)$ such that

$$
\begin{aligned}
-\Delta u(x, y) & =\xi f(x, y) & & \text { in } D, \\
u(x, y) & =0 & & \text { on } \partial D,
\end{aligned}
$$


where $f(x, y):=-432 x(x-1) y(y-1)$ and $\xi \sim \operatorname{Beta}(2,6)$. The QoI is defined by

$$
Q:=\int_{D} u(x, y) d x d y
$$

It is important to note that this problem is a true benchmark problem, for which the exact expected value of $Q$ is given by

$$
\mathbb{E}[Q]=\frac{1}{4} \int_{D} u_{1}(x, y) d x d y
$$

where $u_{1}$ is the solution of the problem for $\xi=1$. Thus only the solution of a single problem on a fine mesh is required to obtain a reference value of $\mathbb{E}[Q]$. This is very useful to verify the implementation and assess the algorithm's performances.

The problem is discretized by finite differences on a sequence of uniform grids and the discretized systems are solved using the NumPy Python package for scientific computing. The source code of the solver itself can be found in

mlmc_routines/ellipt_2d/solver_ellipt.py.

Changing the discretization of the problem or the solver is easily possible and does not require any changes in the used MLMC strategy.

\section{Example of usage}

Before running the demonstrator code, the following packages need to be installed on the system: Python 2 and the appropriate NumPy version. The demonstrator code may then be run by executing the attached file main_ellipt.py (cf. Figure 5).

A possible output in the terminal is presented in Table 1. It shows the evolution of the number of samples on each level during nine adaptation steps. As desired, the algorithm put more samples, and hence computational work, on coarser grids, whereas only a small amount of samples was distributed to finer grids.

\begin{tabular}{c||c|c|c|c|c|c|c|c} 
& $L=1$ & $L=2$ & $L=3$ & $L=4$ & $L=5$ & $L=6$ & $L=7$ & $L=8$ \\
\hline \hline $\mathbf{1}$ & 25 & 25 & 25 & 0 & - & - & - & - \\
\hline $\mathbf{2}$ & 25 & 25 & 25 & 6 & 0 & - & - & - \\
\hline $\mathbf{3}$ & 36 & 25 & 25 & 6 & 6 & 0 & - & - \\
\hline $\mathbf{4}$ & 47 & 25 & 25 & 6 & 6 & 6 & 0 & - \\
\hline $\mathbf{5}$ & 83 & 25 & 25 & 6 & 6 & 6 & 6 & - \\
\hline $\mathbf{6}$ & 307 & 25 & 25 & 6 & 6 & 6 & 6 & - \\
\hline $\mathbf{7}$ & 313 & 25 & 25 & 6 & 6 & 6 & 6 & - \\
\hline $\mathbf{8}$ & 403 & 31 & 25 & 6 & 6 & 6 & 6 & - \\
\hline $\mathbf{9}$ & 493 & 37 & 25 & 6 & 6 & 6 & 6 & 0
\end{tabular}

Table 1: Number of samples per level $L$ adaptively chosen in nine steps.

Depending on the used MLMC strategy and solver, additional output details may be available. These may be helpful in the analysis of the efficiency of the MLMC strategy. In this particular demonstration, the following files are written to the file system: The file 
simulation_io/reports/P1 contains estimated errors and fitted rates for the C-MLMC algorithm, whereas the file simulation_io/reports/P1_val contains the obtained values of the required statistics of the QoI at each C-MLMC iteration.

\section{References}

[1] K. A. Cliffe, M. B. Giles, R. Scheichl, and A. L. Teckentrup. Multilevel monte carlo methods and applications to elliptic pdes with random coefficients. Computing and Visualization in Science, 14(1):3, 2011.

[2] N. Collier, A.-L. Haji-Ali, F. Nobile, E. von Schwerin, and R. Tempone. A continuation multilevel Monte Carlo algorithm. BIT Numerical Mathematics, 55(2):399-432, 2015.

[3] D. Drzisga, B. Gmeiner, U. Rüde, R. Scheichl, and B. Wohlmuth. Scheduling massively parallel multigrid for multilevel Monte Carlo methods. SIAM Journal on Scientific Computing, 39(5):S873-S897, 2017.

[4] D. Elfverson, F. Hellman, and A. Målqvist. A multilevel Monte Carlo method for computing failure probabilities. SIAM/ASA Journal on Uncertainty Quantification, 4(1):312-330, 2016.

[5] M. B. Giles. Multilevel Monte Carlo path simulation. Operations Research, 56(3): 607-617, 2008.

[6] M. B. Giles. Multilevel Monte Carlo methods. Acta Numerica, 24:259-328, 2015.

[7] A.-L. Haji-Ali, F. Nobile, E. von Schwerin, and R. Tempone. Optimization of mesh hierarchies in multilevel Monte Carlo samplers. Stoch. PDEs: Anal. \& Comp., 4(1): 76-112, 2016.

[8] M. Pisaroni, S. Krumscheid, and F. Nobile. Quantifying uncertain system outputs via the multilevel Monte Carlo method - Part I: Central moment estimation. MATHICSE Technical Report 23.2017, École Polytechnique Fédérale de Lausanne, 2017.

[9] M. Pisaroni, F. Nobile, and P. Leyland. A Continuation Multi Level Monte Carlo (CMLMC) method for uncertainty quantification in compressible inviscid aerodynamics. Computer Methods in Applied Mechanics and Engineering, 326:20-50, 2017.

\section{A Snippets}




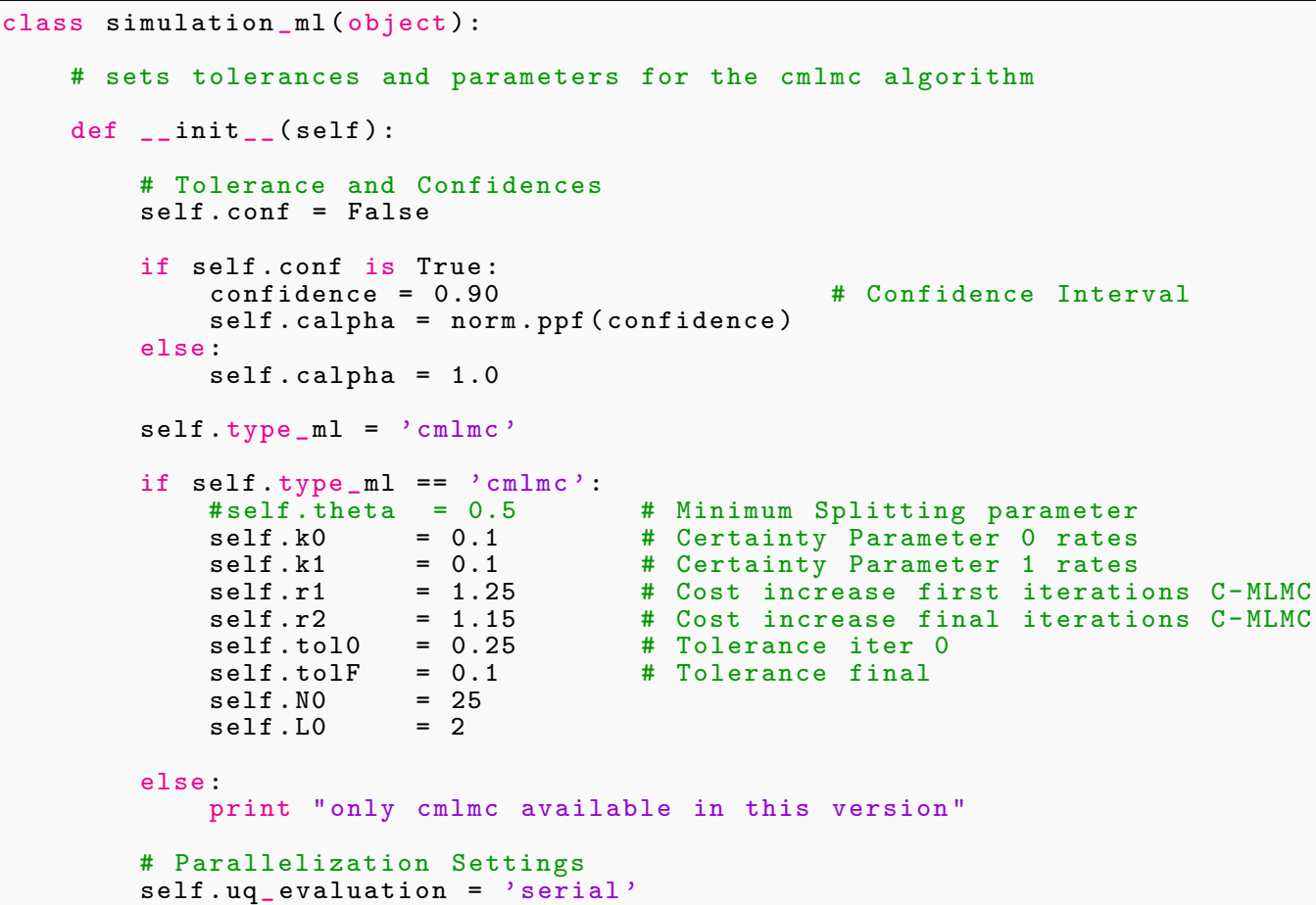

Figure 2: Example MLMC settings class encapsulating all the required MLMC parameters for a particular MLMC strategy. In this case the C-MLMC algorithm.

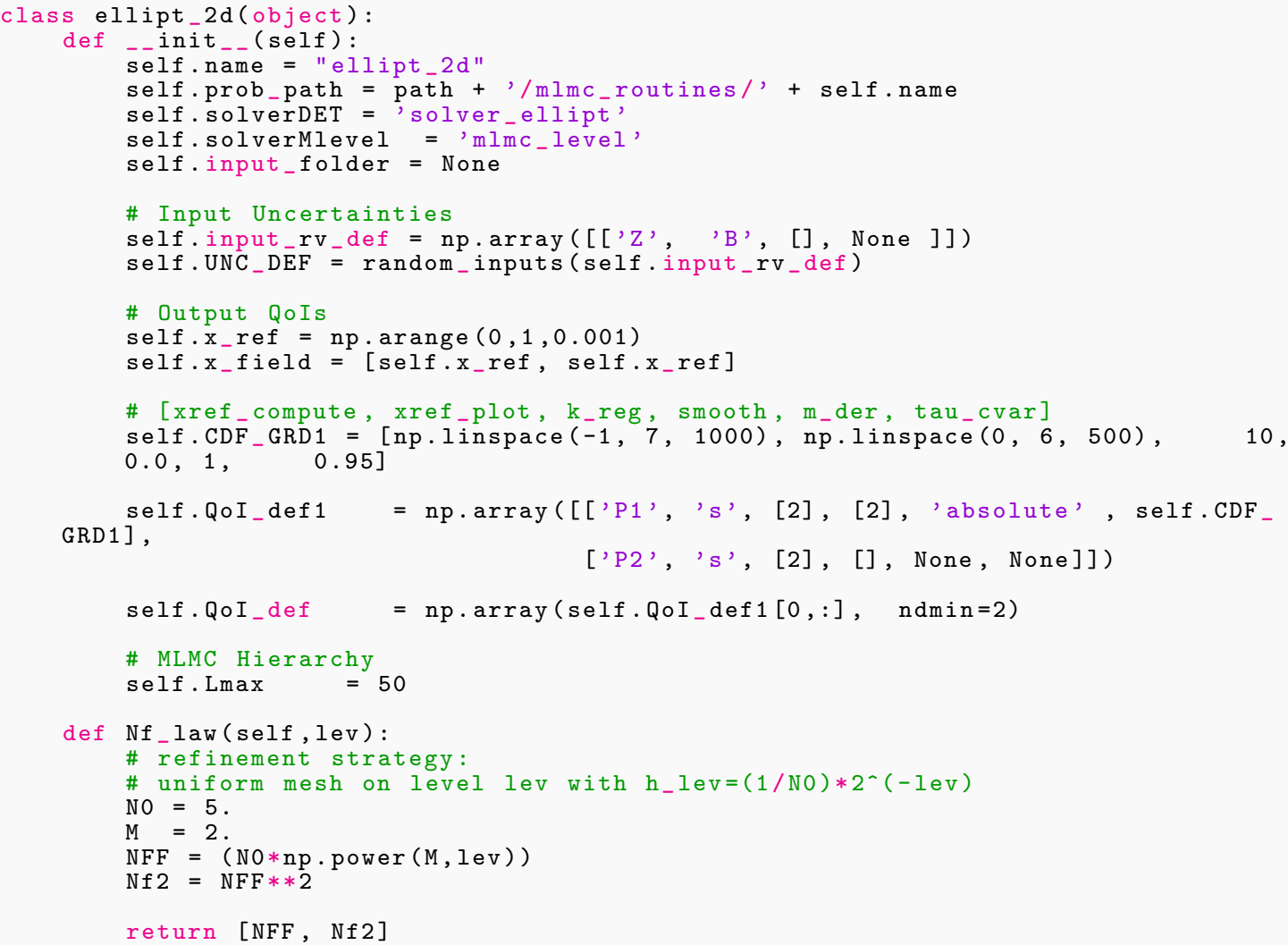

Figure 3: Example implementation of the problem interface. It encapsulates information of the problem, the solver, as well as the QoI. 


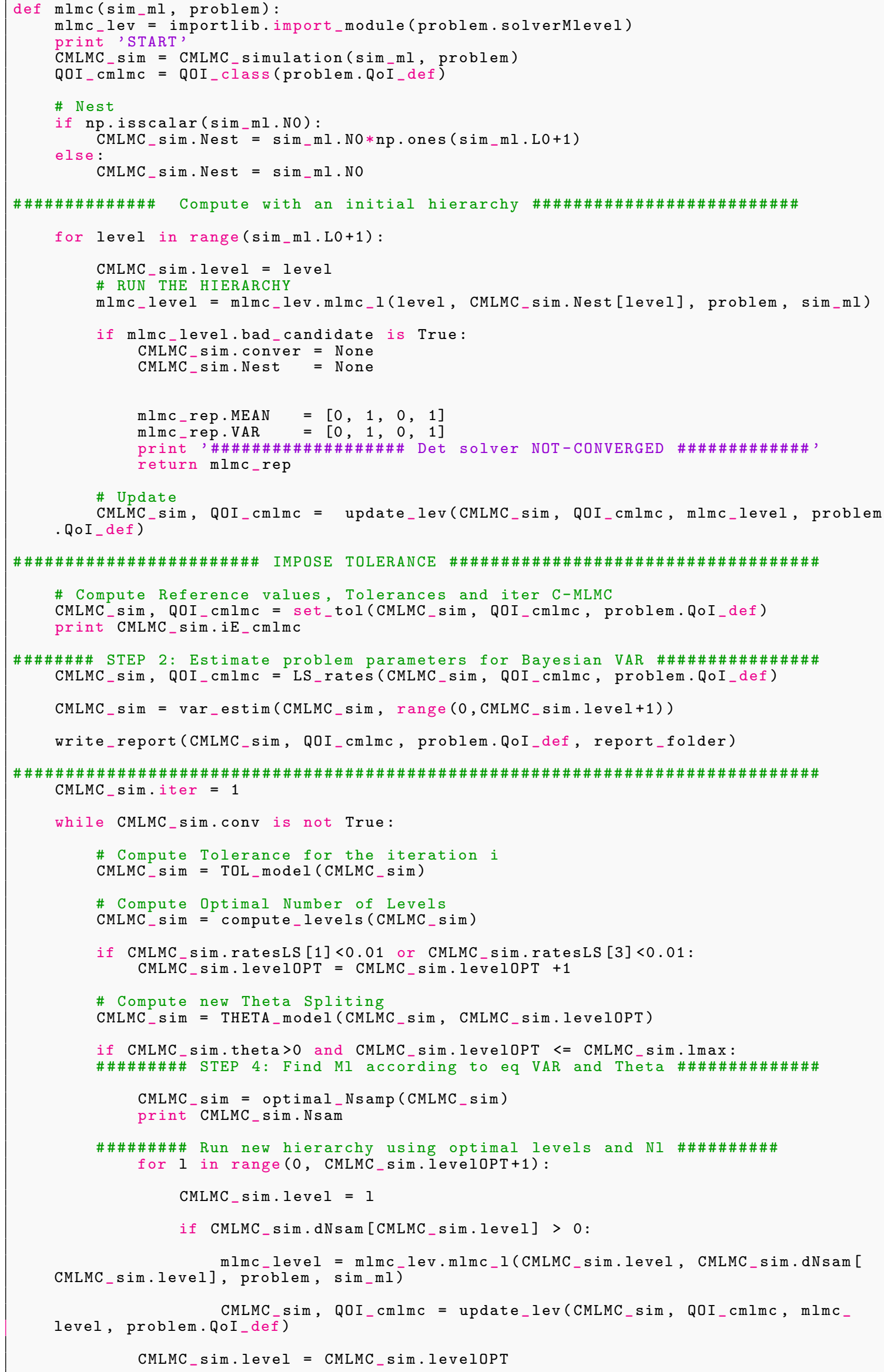

Figure 4: Example implementation of the MLMC core interface. In this particular case, the C-MLMC algorithm is implemented. (part 1) 


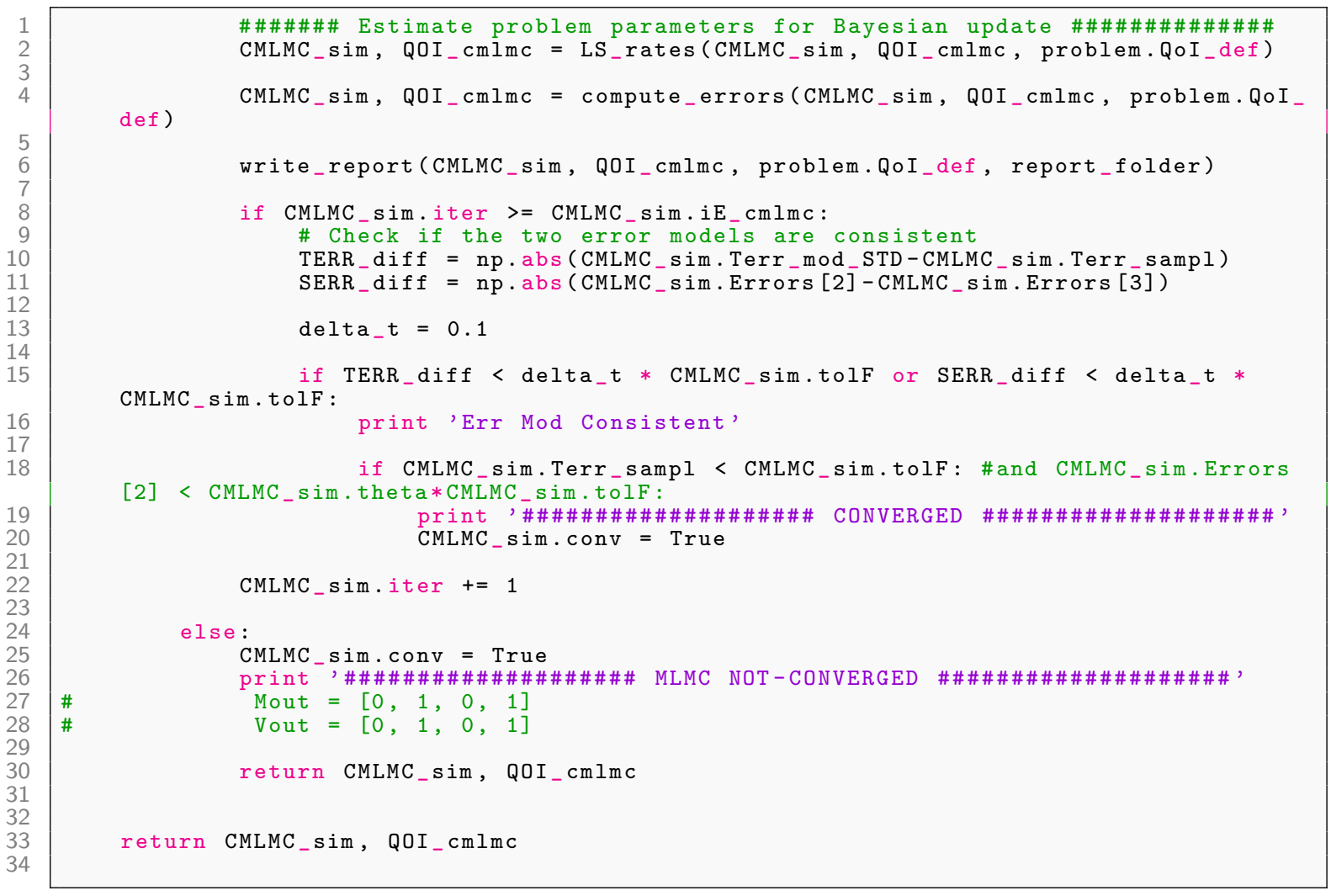

Figure 4: Example implementation of the MLMC core interface. In this particular case, the C-MLMC algorithm is implemented. (part 2)

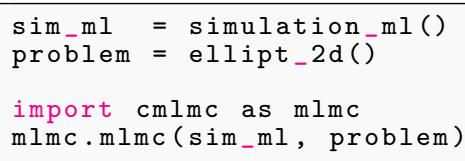

Figure 5: Example program where an MLMC settings and a Problem class are instantiated and the MLMC algorithm is executed. The MLMC algorithm internally instantiates problem solver processes with respective mesh resolutions depending on the underlying MLMC strategy. In this particular example, the C-MLMC algorithm is used. 